\title{
Síndromes de hiperdrenaje de las válvulas en hidrocefalia infantil
}

\author{
J.F. Martínez-Lage; M.A. Pérez-Espejo; M.J. Almagro; J. Ros de San Pedro; F. López; C. Piqueras y J. Tortosa
}

Servicio Regional de Neurocirugía Hospital Universitario Virgen de la Arrixaca. El Palmar. Murcia. Grupo NYNE de Neurología y Neurocirugía Experimental. Facultad de Medicina, Campus de Espinardo. Murcia

Resumen

El drenaje excesivo de las válvulas constituye una complicación dificil, tanto de prevenir como de tratar. Los autores revisan una serie de 512 niños operados mediante derivación de líquido cefalorraquídeo, de manera retrospectiva, para analizar las causas de esta complicación. La causa de la hidrocefalia fue congénita $(n=172)$, debida a mielomeningocele $(n=123)$, posthemorrágica $(n=103)$, tumoral $(n=64)$, postinfecciosa $(n=40)$ y postraumática $(n=10)$. Ochenta y ocho niños $(17,8 \%)$ presentaron alguna complicación por funcionamiento excesivo del sistema valvular. Se analizó la etiología de la hidrocefalia relacionándola con los cuadros de hiperdrenaje. La complicación más frecuente fue la obstrucción del extremo ventricular de la derivación ( $n=$ 50), seguida del cuadro de colapso ventricular sintomático $(n=19)$, de hematoma subdural $(n=10)$ y de cuarto ventrículo aislado $(\mathrm{n}=9)$. Las diversas complicaciones se distribuyeron de manera homogénea entre todos los grupos etiológicos, sin diferencia estadística. El cuadro de colapso ventricular sintomático (CVS) ocurrió en 19 niños $(3,71 \%)$, cifra menor que la descrita en la bibliografía. Sólo dos grupos de pacientes con hidrocefalia adquirida (postinfecciosa y posthemorrágica) tuvieron una mayor tasa de CVS $(p=0,005)$, lo que se atribuyó a la mayor incidencia en estos niños de procesos destructivos cerebrales. El tratamiento del CVS fue complejo y requirió diversos procedimientos, que se aplicaron de manera escalonada, incluyendo cinco craniectomías descompresivas. Los autores recomiendan evitar, en lo posible, el empleo de válvulas, utilizando en su lugar, y siempre que sea posible, técnicas de neuroendoscopia, o válvulas de nueva tecnología, que supuestamente disminuirán el número de complicaciones de hiperfunción valvular.

PALABRAS CLAVE: Hidrocefalia. Colapso ventricular. Hiperfunción valvular. Hiperdrenaje ventricular. Edema

Recibido: 13-09-04. Aceptado: 14-10-04 cerebral. Derivaciones de LCR. Complicaciones de las válvulas de hidrocefalia

Syndromes of overdrainage of ventricular shunting in childhood hydrocephalus

Summary

Overdrainage in ventricular shunting constitutes a difficult to prevent and to treat complication. The authors reviewed a retrospective series of 512 children submitted to a ventricular shunting procedure aimed at analysing factors influencing this type of complication. The causes for the hydrocephalus were congenital $(n=172)$, post-myelomeningocele $(n=123)$, posthemor-rhagic $(n=103)$, tumoral $(n=64)$, postmeningitis $(n=40)$ and posttraumatic $(n=10)$. Eighty-eight children $(17.8 \%)$ evolved with a complication related to the excessive function of the valve. The authors investigated the relationship between hydrocephalus' etiology and type of overdrainage syndromes. The most frequent complication was ventricular catheter block $(\mathbf{n}=\mathbf{5 0})$, followed by symptomatic slit ventricle syndrome (SVS) $(n=19)$, subdural hematoma $(n=10)$ and trapped fourth ventricle $(n=9)$. There were no statistical differences regarding complications for each etiologic subset of hydrocephalus. SVS occurred in 19 children $(3,71 \%)$, a low rate according to the current literature. Posthemorrhagic and postinfectious hydrocephalus grouped together showed a higher rate of SVS $(p=0,005)$, a feature that we attributed to the cerebral destruction caused by these two conditions. Treatment of SVS was complex and required diverse procedures, applied in an escalated way, which included five decompressive craniectomies. The authors suggest avoiding, as much as possible, the use of ventricular shunts, and recommend the alternative use of new technology valves and neu-

Abreviaturas. CVS: colapso ventricular sintomático. DVP: derivación ventrículo-peritoneal. PIC: presión intracraneal. RM: resonancia magnética. TAC: tomografía axial computarizada. 


\section{roendoscopic procedures.}

KEY WORDS: Hydrocephalus. Slit ventricle syndrome. CSF overdrainage. Ventricular overdrainage. Cerebral edema. CSF shunts. Complications of hydrocephalus valves

\section{Introducción}

Durante las últimas cinco décadas el tratamiento de la hidrocefalia ha consistido, fundamentalmente, en la colocación de una derivación de líquido cefalorraquídeo (LCR). El procedimiento usado con mayor frecuencia ha sido la derivación ventrículo-peritoneal (DVP). La técnica quirúrgica de la colocación de la DVP es relativamente sencilla $\mathrm{y}$, con frecuencia su realización se ha relegado a los residentes. Sin embargo, el empleo de las válvulas para la hidrocefalia se ha visto plagado por una multitud de complicaciones, algunas de ellas muy graves. A pesar de las diversas innovaciones tecnológicas, tanto en la construcción como en las propiedades funcionales, de las válvulas, éstas siguen presentando inconvenientes. Una de estas complicaciones, difícil de prevenir y tratar, consiste en el funcionamiento deficiente o excesivo de las válvulas.

En este trabajo, analizamos un grupo de pacientes pediátricos con hidrocefalia, tratados mediante el empleo de una válvula, que presentaron síntomas y signos de funcionamiento excesivo. El objetivo del estudio es analizar la epidemiología de los factores que influyen en la producción de los cuadros de hiperfunción valvular, su patogenia y las diversas opciones propuestas para su tratamiento y prevención.

\section{Pacientes y métodos}

Se revisaron, de manera retrospectiva, los datos demográficos y clínicos correspondientes a 512 niños que recibieron una válvula para el tratamiento de su hidrocefalia en el período de 15 años comprendido entre 1985 a 1999 (ambos inclusive). Se analizaron las etiologías de la hidrocefalia, y se buscaron datos clínicos y neurorradiológicos indicativos de funcionamiento excesivo de la válvula. Los pacientes se clasificaron en 6 subgrupos dependiendo de la etiología de la hidrocefalia: congénita, secundaria a mielomeningocele, tumoral, posthemorrágica, postinfecciosa, y postraumática. La hidrocefalia congénita incluyó casos que son excluidos normalmente en otras series, como malformación de Dandy-Walker, hidrocefalia asociada al quiste aracnoideo, y casos de niños con malformaciones cerebrales complejas. El subgrupo de hidrocefalia posthemorrágica recoge, en su mayoría, un conjunto de niños con ventriculomegalia por hemorragia ventricular, casi todos prematuros. Los pacientes cuyos datos, clínicos o quirúrgicos, fueron incompletos
2005; 16: 124-133

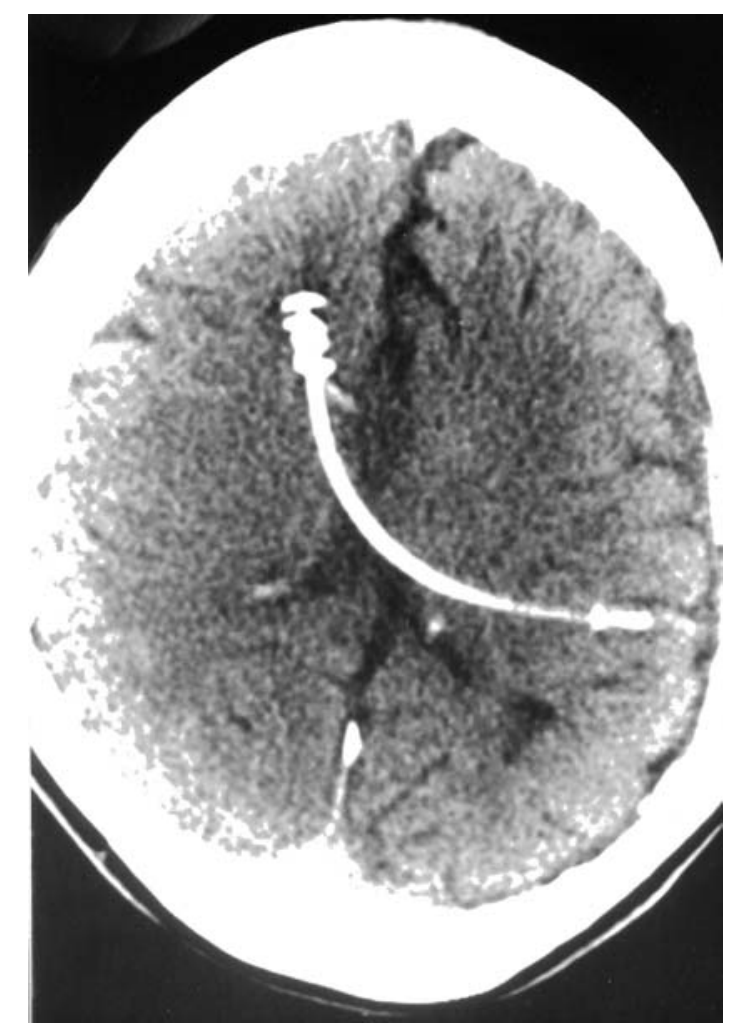

Figura 1. TAC cerebral de un niño operado a los 6 años de hidrocefalia comunicante, mostrando engrosamiento del cráneo y colapso ventricular. El catéter ventricular estaba obstruido.

$(\mathrm{n}=38)$ se excluyeron de la revisión.

Las manifestaciones de hiperfunción valvular se agruparon en cuatro grupos: (a) obstrucción proximal, (b) síndrome de colapso ventricular sintomático (CVS), (c) hematoma subdural, y (d) $4^{\circ}$ ventrículo aislado. El concepto del síndrome de CVS se refiere a la aparición de cefaleas, vómitos, y diferentes grados de alteración de la conciencia, que se acompañan de un relleno lento del reservorio valvular y de la presencia de ventrículos muy pequeños en los estudios de neuroimagen (Fig. 1 y 2). No se incluyeron como casos de hiperdrenaje, los que tenían ventrículos pequeños en la tomografía axial computarizada (TAC) o en la resonancia magnética (RM), si estaban asintomáticos. El término de hematoma subdural denota cualquier colección de LCR o sangre en el espacio subdural que produzca manifestaciones clínicas. Los casos de obstrucción proximal son aquellos en los que se demostró la oclusión, funcional o morfológica, del extremo craneal del sistema de derivación, habitualmente el catéter ventricular. Resulta evidente, que no siempre la obstrucción proximal se debe a drenaje excesivo, pero resultó imposible la discriminación de los casos. Igualmente, la presencia de un $4^{\circ}$ aislado (Fig. 3) se incluyó, entre las complicaciones 


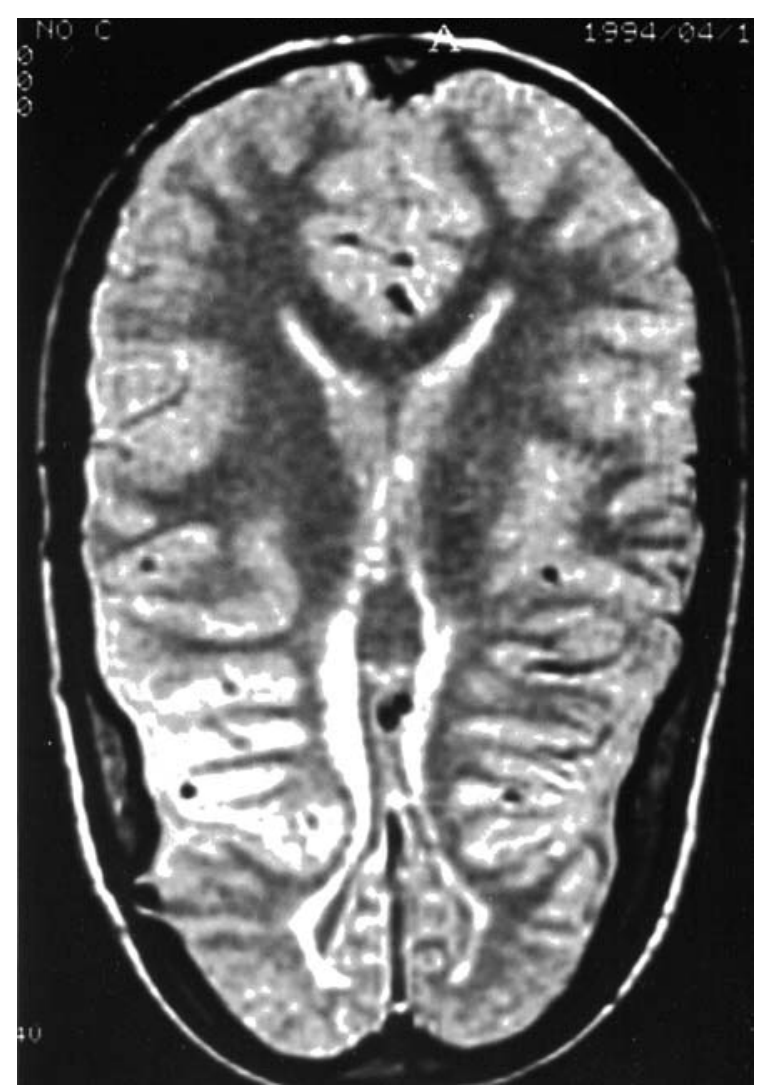

Figura 2. RNM en un niño operado al nacer, de hidrocefalia malformativa, los ventrículos están colapsados y tiene una marcada dolícocefalia.

por hiperfunción, aunque esta eventualidad puede también deberse a otras causas, como es la de la propia etiología de la hidrocefalia.

Se anotaron las características de las válvulas utilizadas, y se registraron los síntomas de presentación, y los resultados de los estudios de neuroimagen. Igualmente, reseñamos las modalidades de tratamiento utilizadas para el manejo de las complicaciones por hiperfunción, y sus resultados. Las comparaciones para validación estadística se realizaron utilizando tablas de 2 × 2 , mediante la prueba de Chi cuadrado y, en el caso de grupos con menos de 5 casos, el test exacto de Fischer (EpiInfo, CDC, USA, vs.5).

\section{Resultados}

La causa más frecuente de hidrocefalia fue la congénita $(\mathrm{n}=172)$, seguida de la asociada a mielomeningocele $(n=123)$, posthemorrágica $(n=103)$, tumoral $(n=64)$, postinfecciosa $(n=40)$, y postraumática $(n=10)$ (Tabla 1). En cuanto a la edad de colocación de la válvula, osciló desde recién-nacido a los 11 años (media 2,8 años). Un 40\% de los niños fue operado en el período neonatal ( 2 prime-

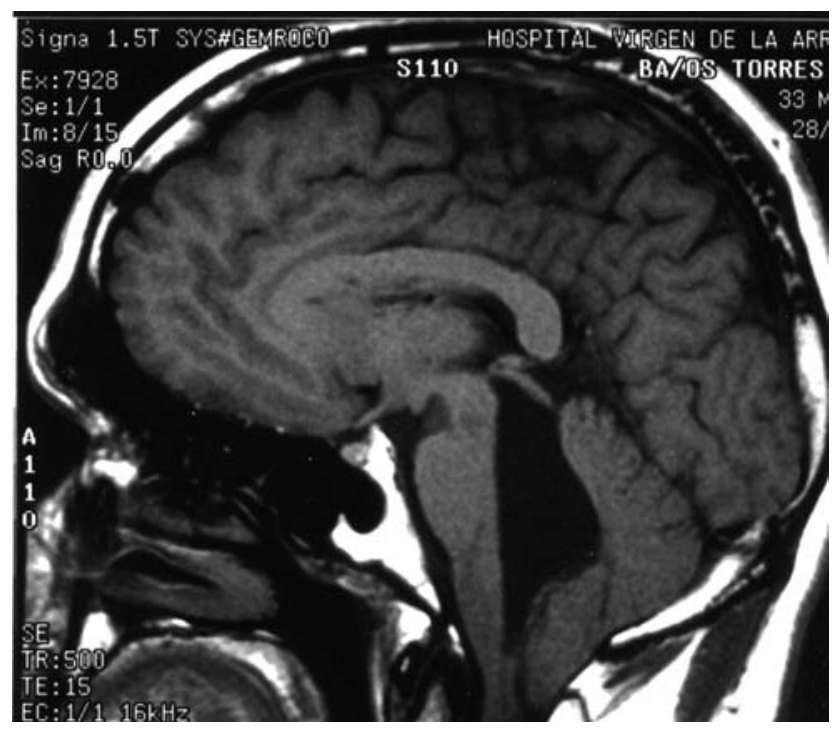

Figura 3. RNM de un adolescente de 14 años, operado de hidrocefalia comunicante en periodo neonatal, mostrando un $4^{\circ}$ ventriculo aislado e inicio de hidromielia cervical.

ros meses de vida), que incluye la mayoría de pacientes con hidrocefalia congénita, posthemorrágica, y debida a mielomeningocele. En esta serie de pacientes pediátricos, todos los niños fueron tratados mediante una DVP de diferentes modelos según la época de inserción y la edad de los niños. Fundamentalmente, se implantaron Unishunts de Raimondi, válvula de Pudenz de 3 piezas, y válvulas de Hakim pediátricas.

La Tabla 1 compara la incidencia de complicaciones por hiperfunción valvular en los seis grupos etiológicos. La tasa de síndromes con drenaje excesivo varió de $0 \%$ en las postraumáticas a $22,5 \%$ en las hidrocefalias postmeningitis, diferencias carentes de significación estadística $(p=0,30)$ dado el pequeño número de niños con hidrocefalia postraumática. Tampoco hubo diferencias significativas en cuanto a número de complicaciones de drenaje excesivo, al comparar todas las hidrocefalias congénitas $(n=295)$, incluyendo los casos de mielomeningocele, con todas las adquiridas $(\mathrm{n}=217)(p=0,52)$. Sin embargo, dos etiologías de las adquiridas, la hidrocefalia posthemorrágica y la postmeningitis, suponen la mayoría de los cuadros de drenaje excesivo, probablemente por tratarse de procesos que comportan mayor destrucción cerebral.

En la Tabla 2 se resumen los cuadros de hiperfunción desglosados por grupos etiológicos. La complicación más frecuente fue la obstrucción del catéter (o el extremo) ventricular de la válvula $(9,76 \%)$, seguida del CVS $(3,71 \%)$, del hematoma subdural $(1,95 \%) \mathrm{y}$, finalmente, del aislamiento del $4^{\circ}$ ventrículo $(1,75 \%)$. La incidencia de los síndromes de hiperfunción valvular fue similar entre los diversos subgrupos etiológicos, observándose, como era de 
Tabla 1

Etiología de la hidrocefalia en 512 niños

\begin{tabular}{lccc}
\hline Etiología & $\begin{array}{c}\text { Total } \\
\mathrm{n}=(\%)\end{array}$ & $\begin{array}{c}\text { Sin hiperfunción } \\
\text { valvular } \mathrm{n}=,(\%)\end{array}$ & $\begin{array}{c}\text { Con hiperfunción } \\
\text { valvular } \mathrm{n}=,(\%)\end{array}$ \\
\hline Congénita & $172(32,95)$ & $143(83,14)$ & $29(16,86)$ \\
Mielomeningocele & $123(23,56)$ & $104(84,55)$ & $19(15,45)$ \\
Posthemorrágica & $103(19,73)$ & $80(77,67)$ & $23(22,33)$ \\
Tumoral & $64(12,26)$ & $56(87,5)$ & $8(12,5)$ \\
Postinfecciosa & $40(7,66)$ & $31(77,5)$ & $9(22,5)$ \\
Postraumática & $10(1,9 \%)$ & $10(100)$ & $-(-)$ \\
TOTALES & $512(100)$ & $424(82,81)$ & $88(17,18)$ \\
\hline
\end{tabular}

Tabla 2.

Complicaciones por drenaje excesivo de las válvulas según etiología

\begin{tabular}{lcccc}
\hline Causa de la hidrocefalia & $\begin{array}{c}\text { Colapso ventricular } \\
\mathrm{n}=(\%)\end{array}$ & $\begin{array}{c}\text { Obstrucción proximal } \\
\mathrm{n}=(\%)\end{array}$ & $\begin{array}{c}4^{\mathrm{o}} \text { aislado } \\
\mathrm{n}=(\%)\end{array}$ & $\begin{array}{c}\text { Colección subdural } \\
\mathrm{n}=(\%)\end{array}$ \\
\hline Congénita & $4(2,32)$ & $16(9,3)$ & $4(2,32)$ & $5(2,9)$ \\
Mielomeningocele & $1(0,98)$ & $17(13,8)$ & $1(0,8)$ & - \\
Posthemorrágica & $8(7,77)$ & $11(10,68)$ & $2(1,94)$ & $2(1,94)$ \\
Tumoral & $1(1,56)$ & $3(4,68)$ & $1(1,56)$ & $3(4,68)$ \\
Postmeningitis & $5(12,5)$ & $3(7,5)$ & $1(2,5)$ & - \\
Postraumática & - & - & - & - \\
TOTAL & $19(3,71)$ & $50(9,76)$ & $9(4,7)$ & $10(1.95)$ \\
\hline
\end{tabular}

esperar, una mayor incidencia del síndrome de CVS en las hidrocefalias adquiridas en comparación con las congénitas $(p=0,005)$.

Los diversos tratamientos empleados se muestran en la Tabla 3. La hiperfunción valvular se presentó en 88 pacientes $(17,19 \%)$ y supuso 101 procedimientos quirúrgicos. La sustitución del catéter ventricular $(\mathrm{n}=27)$ y el cambio a válvulas de mayor presión o a derivaciones con diferente característica de flujo (reguladas por flujo o con dispositivo antisifón incorporado) $(\mathrm{n}=37)$ y el cambio a válvula programable $(n=25)$ fueron los tratamientos empleados con más frecuencia (en conjunto, 89 ó $87,25 \%$ de todas las intervenciones). La alta tasa de sustituciones de toda la derivación supone el $60,78 \%$ de todos los tratamientos de la hiperfunción, y se realizó pensando que el origen del cuadro de drenaje excesivo dependía precisamente de las características de presión/flujo de la válvula. En los primeros años del estudio se realizaron cambios de derivación a sistemas de mayor presión, o de mecanismo distintos al de diferencia de presión, mientras que en los últimos cuatro años la sustitución se realizó preferentemente con válvulas programables (Sophysa, Francia).

La complicación de hematoma subdural se produjo en 10 niños, que fueron tratados mediante cambio de válvula en $10 \mathrm{y}$, además, mediante trépano y drenaje subduroperitoneal en dos. El tratamiento del $4^{\circ}$ ventrículo aislado se presentó en 4 casos, y se corrigió mediante colocación de otra válvula cuarto ventrículo-peritoneal. A pesar de ello, tres niños requirieron craniectomías descompresivas de fosa posterior por síndrome de $4^{\circ}$ ventrículo atrapado asociado a descenso de las amígdalas cerebelosas. Para el tratamiento del síndrome de CVS sintomático (29 casos) se requirieron los siguientes procedimientos: 13 cambios del tipo/presión de la válvula, 14 cambios a válvula programable y 2 craniectomías descompresivas.

El tiempo de seguimiento osciló entre los 3 y 19 años 
Tabla 3

Procedimientos empleados en 88 pacientes con complicaciones por drenaje excesivo de las válvulas

\begin{tabular}{lccccccc}
\hline Etiología & $\begin{array}{l}\text { Cambio } \\
\text { de catéter } \\
\text { ventricular }\end{array}$ & $\begin{array}{l}\text { Trépano } \\
+ \text { DSP }\end{array}$ & $\begin{array}{l}\text { Doble } \\
\text { válvula }\end{array}$ & $\begin{array}{l}\text { Cambio de } \\
\text { presión o tipo } \\
\text { de válvula }\end{array}$ & $\begin{array}{l}\text { Inserción } \\
\text { de válvula } \\
\text { programable }\end{array}$ & $\begin{array}{c}\text { Craniectomía } \\
\text { descompresiva }\end{array}$ & TOTAL \\
\hline Congénita & 6 & 1 & 2 & 13 & 9 & 1 & 32 \\
MMC & 6 & - & - & 9 & 4 & 1 & 20 \\
Posthemorrágica & 9 & - & - & 7 & 7 & - & 1 \\
Tumoral & 3 & 1 & 2 & 4 & 3 & 23 \\
Postmeningitis & 3 & - & - & 4 & 2 & 2 & 11 \\
TOTALES & 27 & 2 & 4 & 37 & 25 & 5 & 101 \\
$(\%)$ & $(30.7 \%)$ & $(2,27 \%)$ & $(4,55 \%)$ & $(42,05 \%)$ & $(28,41 \%)$ & $(5,68 \%)$ & \\
\hline
\end{tabular}

$\mathrm{DSP}=$ drenaje subdural-peritoneal, MMC mielomeningocele

(promedio 6,5). No se produjo ninguna muerte en relación con el tratamiento quirúrgico de estos niños, si bien en el grupo de hidrocefalia tumoral se produjeron 16 defunciones por causas relacionadas con el proceso neoplásico que motivó la hidrocefalia.

\section{Discusión}

Sindromes de funcionamiento excesivo de las válvulas de hidrocefalia.

Existen una serie de manifestaciones clínicas que se han relacionado con el drenaje excesivo y prolongado de LCR. Estos cuadros son: 1. Cefaleas ortostáticas, que se presentan en ciertos pacientes, casi siempre de manera transitoria, y que se deben a hipotensión intracraneal durante la bipedestación ${ }^{12,14}$. 2. Cambios óseos craneales, como engrosamiento y laminación de la bóveda craneal, esclerosis de las suturas, estrechamiento y remodelación de los agujeros de la base craneal, y dilatación de los senos paranasales ${ }^{20,36} .3$. Microcefalia y craniosinostosis: muchos niños con drenaje prolongado de LCR presentan microcefalia, sobre todo en casos de daño cerebral importante, como sucede en la hidrocefalia posthemorrágica y postinfecciosa. La microcefalia se debe a la falta de impulso cerebral que lleva a una detención del crecimiento craneal. Se ha publicado algún caso de craniosinostosis auténtica en niños hidrocefálicos portadores de válvulas ${ }^{1,24}$. 4. Hematomas e higromas subdurales: se deben a desproporción craneoencefálica, pueden presentarse de manera aguda, subaguda o crónica, sobre todo cuando las suturas están ya cerradas ${ }^{9,42} .5$. Cambios del propio cerebro y meninges, como invaginación de la corteza, engrosamiento meníngeo y aracnoideo, surcos ensanchados, distorsión del tronco cerebral y de los nervios oculomotores y quistes aracnoideos secundarios ${ }^{23,26,43}$. 6 . Tabicamientos o aislamiento de los ventrículos laterales y $4^{\circ}$ ventrículo atrapado: se han descrito como consecutivos al drenaje excesivo de LCR, aunque en su patogenia parece haber implicados otros factores de tipo inflamatorio, como en las hidrocefalias postmeningitis, postraumáticas y posthemorrágicas ${ }^{13,17,30,32,44}$. 7. Desproporción craneoencefálica: esta condición se refiere a un mayor crecimiento cerebral respecto al craneal, pudiendo producir "apiñamiento" de las estructuras de la fosa posterior a nivel del agujero magno ${ }^{6,18,33,39} .8$. Estenosis del canal lumbar: esta complicación no sólo se ha descrito en casos de derivación lumbo-peritoneal, sino también tras las DVP ${ }^{22}$.

Patogenia del síndrome de colapso ventricular sintomático (CVS)

Existe un acuerdo mayoritario en que la causa del CVS reside en un drenaje excesivo de LCR desde los ventrículos en los pacientes portadores de derivaciones ventriculares ${ }^{8,35,40}$. Este cuadro se produce tras un período variable de tiempo de funcionamiento normal de la derivación. Varios factores contribuyen a su producción, ya que se presenta más frecuentemente en niños operados de muy pequeña edad, en pacientes tratados con una válvula de presión baja y en los operados de hidrocefalia posthemorrágica o postmeningítica. El resultado del drenaje excesivo y prolongado de LCR desde los ventrículos produce: una disminución del tamaño ventricular, una reducción de los espacios subaracnoideos, disminución de la presión intraventricular $y$, sobre todo, reducción de la amplitud de la presión de pulsación cerebral. El crecimiento craneal 
Tabla 4.

Tipo y número de intervenciones para el tratamiento de 88 niños con síndromes de hiperfunción valvular

\begin{tabular}{|c|c|c|c|c|c|c|c|}
\hline & $\begin{array}{l}\text { Cambio } \\
\text { catéter } \\
\text { ventricular }\end{array}$ & $\begin{array}{l}\text { Trépano } \\
+ \text { DSP }\end{array}$ & $\begin{array}{l}\text { Doble } \\
\text { válvula }\end{array}$ & $\begin{array}{l}\text { Sustitución de } \\
\text { toda la } \\
\text { válvula }\end{array}$ & $\begin{array}{l}\text { Inserción } \\
\text { válvula } \\
\text { programable }\end{array}$ & Craniectomías & TOTAL \\
\hline Obstrucción & 27 & - & - & 17 & 6 & - & 50 \\
\hline \multicolumn{8}{|c|}{ Catéter proximal } \\
\hline $\begin{array}{l}\text { Hematoma } \\
\text { subdural }\end{array}$ & - & $2(*)$ & - & $6(*)$ & $4(*)$ & - & 12 \\
\hline $4^{\circ}$ aislado & - & - & 4 & $1(*)$ & 1 & 3 & 10 \\
\hline $\begin{array}{l}\text { Colapso } \\
\text { ventricular }\end{array}$ & - & - & - & 13 & 14 & 2 & 29 \\
\hline TOTALES & 27 & 2 & 4 & 37 & 25 & 5 & 101 \\
\hline
\end{tabular}

Tabla 5

Incidencia de colapso ventricular en varias series (modificado de Di Rocco, ref. 8)

\begin{tabular}{lcccc}
\hline Autor(es) & Seguimiento (años) & $\mathrm{N}^{\text {o }}$ pacientes & Pacientes con colapso ventricular & $\%$ \\
\hline Keucher \& Mealey & 12 & 228 & 3 & 1,3 \\
Oi \& Matsumoto & - & 164 & 5 & 3 \\
Mc Laurin \& Olivi & 7 & - & 15 & $3-5$ \\
Epstein & 17 & - & 20 & - \\
Serlo & 19 & 141 & 52 & 37 \\
Choux & 18 & - & 28 & 0,9 \\
Benzel & 5 & 92 & 22 & 24 \\
Di Rocco & 17 & 625 & 6 & 0,9 \\
Este trabajo & 19 & 512 & 19 & 3,7 \\
\hline
\end{tabular}

normal, requiere el impulso continuo del cerebro en crecimiento. A su vez, la expansión craneal es el resultado de un volumen cerebral creciente, cuyo motor es la presión ventricular y la presión del pulso cerebral. Estos estímulos se ven amortiguados cuando se coloca una válvula, lo que ocasiona una rigidez del cerebro y sus cubiertas. La falta de elasticidad craneal produce engrosamiento de la bóveda (y probablemente de la base) craneal así como esclerosis precoz de las suturas craneales ${ }^{36}$. Otro factor importante parece ser que las suturas y fontanelas estén abiertas en el momento de la colocación de la válvula. El CVS rara vez se presenta en adolescentes o adultos. Esta falta de elasticidad craneal se transmite a la duramadre y probablemente a los senos venosos. La dificultad al flujo del LCR también se ha visto implicada en la patogenia del síndrome de CVS. Las paredes ventriculares también pueden volverse rígidas por gliosis ${ }^{7}$. Por todos estos factores, el cerebro queda "apretado" a la vez que es comprimido dentro del cráneo. Esta situación es similar a los cuadros de hipertensión intracraneal que se ven en el pseudotumor cerebri y en ciertos casos de craniosinostosis ${ }^{7,27}$. Los cambios cerebrales ya han sido mencionados en el epígrafe anterior (reducción ventricular, engrosamiento de la aracnoides, etc. $)^{9}$. En resumen, el factor desencadenante del síndrome parece consistir en la compresión del cerebro en desarrollo ocasionada por las cubiertas meníngeas y craneales que han perdido su elasticidad.

\section{Epidemiología del CVS}

La frecuencia exacta del síndrome de CVS es descono- 


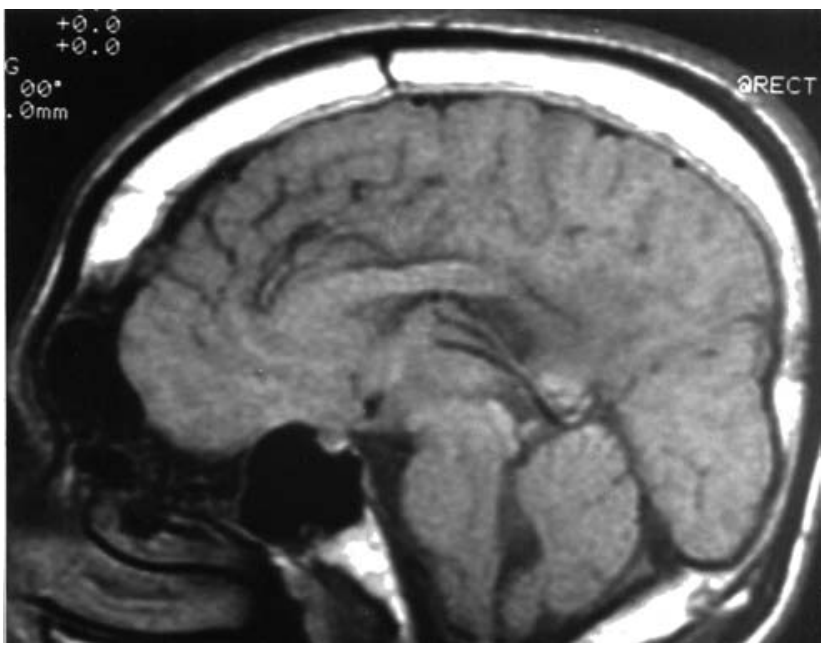

Figura 4. RNM en un niño de 12 años, operado al mes de vida de hidrocefalia posthemorrágica por prematuridad. Nótese el engrosamiento craneal, la permeabilidad de las suturas y el pequeño tamaño de la fosa posterior, con apiñamiento de estructuras cerebrales

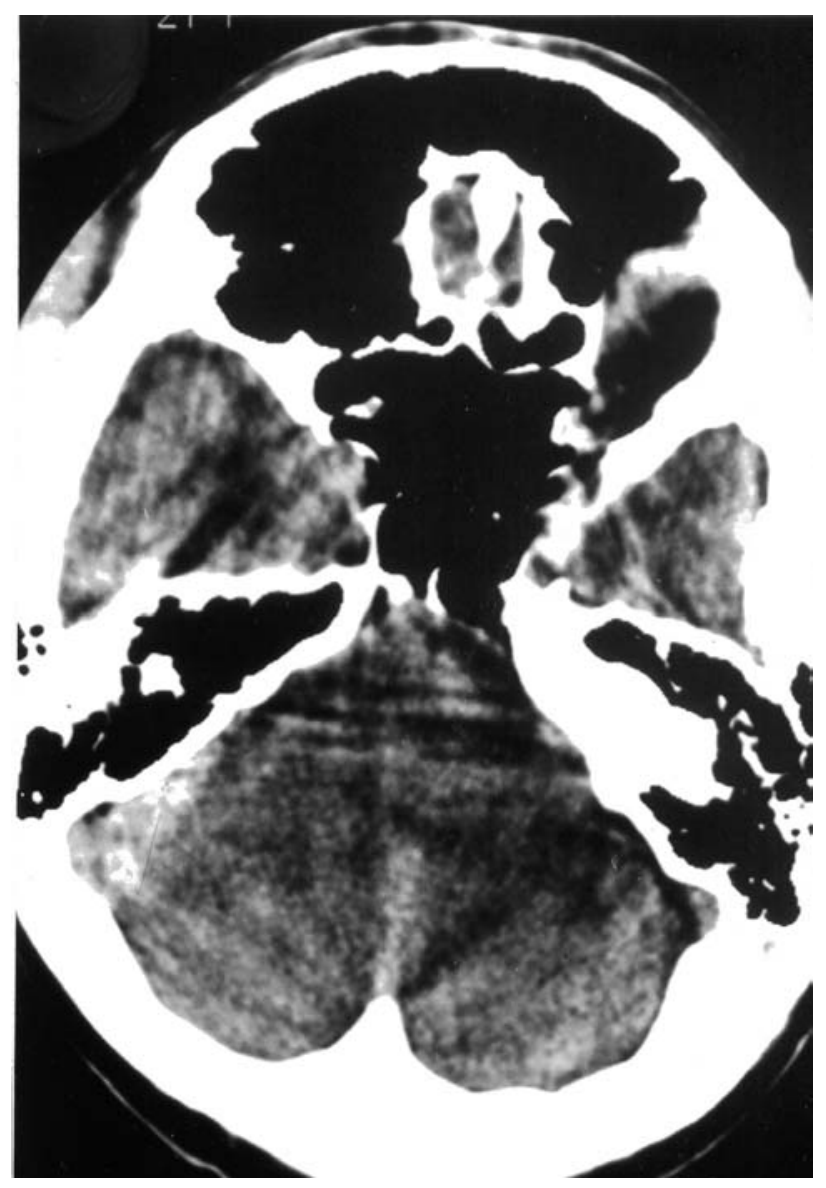

Figura 5. TAC cerebral en un caso de colapso ventricular sintomático mostrando insuflación de senos paranasales y celdas mastoideas

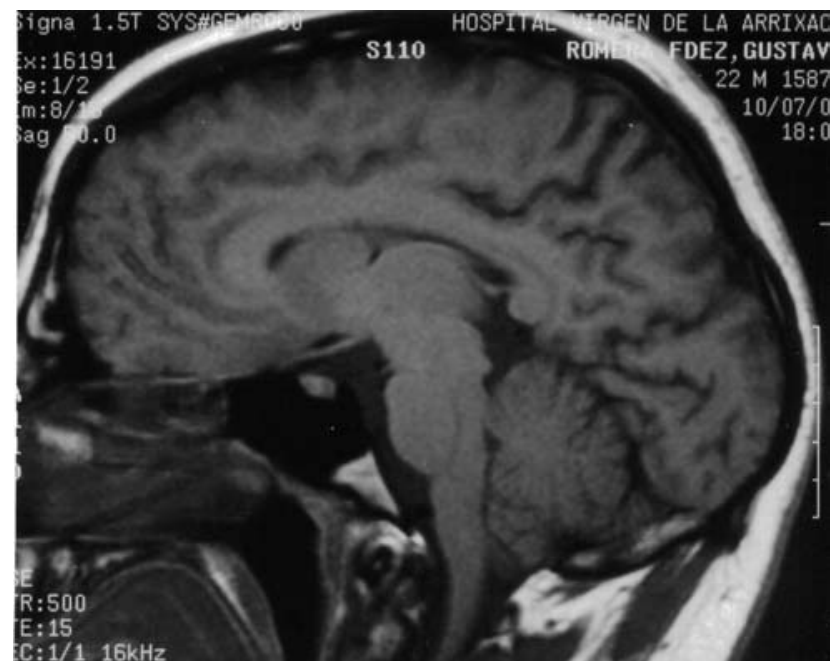

Figura 6. RNM en un niño de 13 años mostrando compresión cerebral e inicio de herniación de las amígdalas cerebelosas

cida, oscilando en diferentes series de $1 \%$ a 37\% $3,4,8,11,21,40$, y en nuestra experiencia, del 3,7\%. La Tabla 5 muestra la incidencia de CVS publicada por varios autores $3,4,8,11,21,40$. Factores como el número de revisiones quirúrgicas de las válvulas y la presión de apertura de la válvula no parecen influir en la incidencia del síndrome de CVS. En varios trabajos, el cuadro predomina en niños tratados con válvulas de presión baja ${ }^{19,36}$, aunque en la serie de Di Rocco todos los niños habían sido tratados con una válvula de presión media ${ }^{8}$. Sólo un estudio mostró una disminución en la incidencia del CVS; se trata de válvulas reguladas por flujo $(\text { Orbis-Sigma })^{38}$. En un trabajo anterior de nuestro hospital, se encontró una relación entre el CVS y la inserción de la válvula a una edad muy temprana ${ }^{36}$. En el presente trabajo, no hemos encontrado ninguna correlación, excepto una mayor incidencia de CVS en casos de hidrocefalia "destructiva" en comparación con las formas congénitas $(p=0,005)$. Igualmente, hemos encontrado una mayor proporción de casos de obstrucción del extremo ventricular de la derivación en pacientes que luego desarrollaron cuadros de CVS.

\section{Clínica del síndrome de colapso ventricular}

Respecto a la edad, la mayoría de los casos del SCV se presentan en la primera década de la vida, existiendo un pico de los 4 a los 7 años, y en nuestra serie, de 6,5 años. El intervalo desde la colocación de la válvula oscila de varios meses a años, tras un período de funcionamiento normal de la derivación ${ }^{8}$. En cuanto a la clínica, el cuadro más característico consiste en cefalea, vómitos y grados variables de afectación de la conciencia. También puede haber papiloedema. De acuerdo con Di Rocco ${ }^{8}$ la diferen- 
cia de la cefalea del CVS con la de la cefalea ortostática estriba en que no está relacionada con la posición y no se alivia al acostarse. Otras manifestaciones clínicas consisten en somnolencia paroxística, ataques, ataxia, y trastornos endocrinológicos ${ }^{8}$. Nosotros hemos notado un aumento del número de crisis comiciales en niños con CVS. Una característica del CVS es la naturaleza intermitente de los síntomas.

\section{Diagnóstico del síndrome de colapso ventricular}

Las radiografías de cráneo presentan desde el inicio del cuadro de CVS: engrosamiento de la bóveda, esclerosis de las suturas, sobre todo de la coronal, y frecuentemente una fosa posterior pequeña y un aumento de las cavidades de los senos paranasales, cambios que también son visibles en la TAC y RM (Fig. 4 y 5). Estos hallazgos son casi constantes y se manifiestan, o empeoran, con el paso del tiempo. La característica más típica del CVS consiste en el hallazgo, en la TAC y en la RM, de ventrículos muy pequeños (Fig. 1 y 2), así como del apiñamiento de estructuras dentro del cráneo y en la fosa craneal posterior ${ }^{8}$, incluso con signos de herniación cerebelosa (Fig. 6). Al igual que los ventrículos, el espacio subaracnoideo de la convexidad se halla prácticamente desaparecido, una situación que se parece mucho a la del edema cerebral. En fases agudas, los ventrículos pueden aumentar ligeramente de tamaño. Un signo de riesgo inminente es la obliteración de las cisternas perimesencefálicas. En el EEG pueden asistir alteraciones focales o difusas de la actividad cerebral.

El valor del relleno lento del reservorio de la válvula después de bombearlo, es un signo muy discutido, aunque a nosotros nos parece fiable ante el adecuado contexto clínico. Es más importante la punción del reservorio, ya que sirve para descartar la existencia de una obstrucción del catéter ventricular. Durante la punción, se puede medir la presión ventricular, que puede ser normal o estar aumentada, siendo más fiable el registro de presión lumbar, o los registros continuos intraparenquimatoso, ventricular o epidural. Los registros continuos de presión intracraneal (PIC) pueden mostrar valores elevados, normales o incluso disminuidos ${ }^{8}$. Rekate empleó el registro continuo de PIC para clasificar el síndrome de CVS en distintos tipos $^{35}$. El test de infusión lumbar normalmente muestra una reabsorción defectuosa de LCR, lo que indica rigidez de las paredes ventriculares. Esto explicaría por qué los ventrículos no se dilatan a pesar de aumentar la PIC. Otros autores notan una elevada elastancia cerebral caracterizada por una alta amplitud de la presión de pulsación ventricular, que no se acompaña de aumento de la PIC. El shuntograma con contraste radio-opaco y el shuntograma isotópico se emplean menos frecuentemente, y sirven para demostrar que la válvula está permeable. Nosotros hemos utilizado en algún caso estudios de SPECT que muestran zonas de hipoperfusión.

\section{Tratamiento del CVS}

Como hemos visto, la patogenia del CVS no está bien aclarada. Se han identificado una serie de situaciones que producen CVS por lo que se precisan diferentes formas de tratamiento $8,10,11,19,31,34,35,37,40,45$. El primer paso debe consistir en descartar la obstrucción del catéter ventricular mediante una punción del reservorio. Si el catéter está obstruido, la solución quirúrgica consiste en cambiarlo, y probablemente sustituir el mecanismo de la válvula por otra con una mayor presión de apertura, para evitar que se repita la obstrucción. La oclusión parcial del catéter ventricular puede hacer muy difícil el diagnóstico de disfunción valvular.

En el CVS existen dos principales opciones de tratamiento quirúrgico:

1. La primera opción consiste en cambiar la válvula por otra de mayor presión de apertura, o en añadir un dispositivo antisifón ${ }^{16,41,45}$. Esta solución funcionó muy bien en nuestros pacientes más pequeños. En los últimos años del estudio, hemos preferido utilizar un tipo de válvula programable (Sophy).

2. La segunda opción quirúrgica está encaminada a aumentar la capacidad craneal. Se han descrito varios tipos de craniectomías descompresivas con este fin, como la descompresión subtemporal del lado del ventrículo que aloja al catéter ventricular ${ }^{10,11,19,45}$. También se han empleado técnicas de las utilizadas para tratamiento de las craneostenosis, como el avance frontal ${ }^{8,11}$. Nosotros hemos utilizado craniectomías biparietales amplias, sin abrir la duramadre. Algunos autores indican que el tamaño ventricular, en la TAC o RM, no varía tras las descompresiones óseas, a pesar de que los pacientes queden asintomáticos ${ }^{8}$.

Se han descrito recientemente nuevas modalidades de tratamiento. Se ha empleado con éxito la ventriculostomía endoscópica del tercero en casos de hidrocefalia obstructiva $^{2,34}$. Reddy et al. han utilizado esta técnica en cinco casos en los que habían fracasado previamente otros tratamientos (válvulas de presión alta, dispositivos antisifón, y craníectomía subtemporal) ${ }^{34}$. Algunos autores recomiendan la retirada definitiva de la válvula en casos de $\mathrm{CVS}^{2,34}$. La filosofía que sustenta esta opción es que algunos casos de CVS representan pacientes que ya no necesitan la válvula. Lundar ${ }^{25}$ utiliza el test de infusión lumbar y Fouyas et al. ${ }^{15}$ registros intraparenquimatosos de PIC para detectar estos pacientes. El método de Baskin et al. ${ }^{2}$ consiste en ligar o retirar la derivación mientras observa al paciente en la UCI. Finalmente, hay que considerar que algunos pacientes con sospecha de tener un cuadro de CVS, tienen cefaleas tipo migraña y deben ser tratados sólo con los fármacos apropiados ${ }^{28,29}$. 


\section{Prevención del cuadro}

Está claro que la mejor válvula es... ninguna válvula. La reintroducción de técnicas de neuroendoscopia sin duda contribuirá a disminuir la necesidad de poner una derivación de LCR, y, por tanto, disminuirán sus complicaciones. Sin embargo, hay que tener en cuenta que ciertos casos no son susceptibles de ventriculostomía, y que, además, la ventriculostomía también puede fracasar. Hemos vivido unos años de contemplar las complicaciones de las válvulas, y en un futuro próximo probablemente presenciaremos las de la neuroendoscopia. Otro camino para la prevención de las complicaciones de hiperdrenaje, lo constituye el empleo de válvulas construidas con nuevas tecnologías, programables, reguladoras del flujo etc. Actualmente, estamos realizando un estudio sobre la utilidad de las válvulas programables en la prevención de estas complicaciones.

\section{Conclusiones}

1. Existen varios síndromes relacionados con el funcionamiento excesivo de las válvulas de hidrocefalia. La caracterización del tipo de síndrome que padece el paciente constituye el primer paso para su tratamiento. El mejor tratamiento es el encaminado a tratar el tipo específico de síndrome de hiperfunción que justifique los síntomas del paciente. 2. En nuestro estudio, la incidencia global de cuadros de funcionamiento excesivo de las válvulas fue del $17,18 \%$ y el del síndrome de colapso ventricular de $3,71 \%$. 3. Se requieren estudios más amplios, quizás multicéntricos, para evaluar los diversos métodos en uso para la prevención y tratamiento de estos síndromes.

\section{Notas y agradecimientos}

Los autores agradecen la colaboración del Sr. Espin, fotógrafo del Hospital, por su gran experiencia y arte en la producción de las fotos. Los autores no tienen ningún interés material en ningún tipo de válvula de las que se mencionan en el texto. Parte de este trabajo se presentó en el $5^{\text {th }}$ European Course in Pediatric Neurosurgery, organizado por la ESPN, en Brasov, Rumania, mayo 2000.

\section{Bibliografía}

1. Anderson, H.: Craniosynostosis as a complication after operation for hydrocephalus. Acta Pediatr Scand 1966; 55: 192-196

2. Baskin, J.J., Manwaring, K.H., Rekate, H.L.: Ventricular shunt removal: the ultimate treatment for slit ventricle syndrome. J Neurosurg 1998; 88: 478-484

3. Benzel, E.C., Reeves, J.D., Kesterson, L., Hadden, T.A.: Slit ventricle syndrome in children: clinical presentation and treatment. Acta Neurochir (Wien) 1992; 117:7-14.

4. Choux, M., Genitori, L.: Slit ventricle syndrome. Intern Symp on Hydrocephalus, Kobe 1990 (citado por ref. 8).

5. Choux, M.: Consensus: treatment. Child's Nerv Syst 1994; 10: 74-75.

6. Churras, P.D., Armstrong, D.C., Drake, J.M., et al.: Tonsillar hermation: the rule rather than the exception after lumboperitoneal shunting in the pediatric population. J Neurosurg 1993; 78: 568-573.

7. Dahlerup, B., Gjerris, F., Haimsen, A., Sorensen, P.S.: Severe headache as the only symptom of long-standing shunt dysfunction in hydrocephalic children with normal or slit ventricles revealed by computed tomography. Child's Nerv Syst 1985; 1: 49-52.

8. Di Rocco, C.: ls the slit ventricle always a slit ventricle syndrome?. Child's Nerv Syst 1994; 10: 49-58.

9. Emery, J.L.: Intracranial effects of long-standing decompression of the brain in children with hydrocephalus and myelomeningocele. Develop Med Child Neurol 1965; 7 : 302-309.

10. Epstein, F., Fleischer, A.S., Hochwald, G.M., Ransohoff, J.: Subtemporal craniectomy for recurrent shunt obstruction secondary to small ventricles. J Neurosurg 1974; 41 : 29-31.

11. Epstein, F., Lapras, C., Wisoff, J.H.: Slit-ventricle syndrome: etiology and treatment. Pediatr Neurosci 1988; 14 : $5-10$.

12. Faulhaur, K.: The overdrained hydrocephalus. Clinical manifestations and management. In Krayenbuhl H: Advances and technical standards in Neurosurgery. Springer, Berlin, 1982; vol. 9, pp 3-24.

13. Foltz, E.L., De Foe, D.R.: Isolated $4^{\text {th }}$ ventricle (or double compartment hydrocephalus): a new clinical entity. Child's Brain 1979; 5: 557.

14. Foltz, E.L., Blanks, J.P.: Symptomatic low intracranial pressure in shunted hydrocephalus. J Neurosurg 1988; 68: 401-408.

15. Fouyas, I.P., Casey, A.T.H., Thompson, D., et al.: Use of intracranial pressure monitoring in the management of childhood hydrocephalus and shunt-related problems. Neurosurgery 1996; 38: 726-732.

16. Gruber, R., Jenny, P., Herzog, B.: Experiences with the anti-siphon device in shunt therapy of pediatric hydrocephalus. J Neurosurg 1984; 61: 156-162.

17. Hawkins, J.C. III, Hoffman, H.J., Humphreys, R.P.: Isolated fourth ventricle as a complication of ventricular shunting. Report of three cases. J Neurosurg 1978; 49: 910-913.

18. Hoffman, H.J., Tucker, W.S.: Cephalocranial disproportion A complication of the treatment of hydrocephalus in children. Child's Brain 1976; 2: 167-176.

19. Holnes, R.O., Hoffman, H.J., Hendrick, E.B.: Subtemporal decompression for slit-ventricle syndrome after shuntng in hydrocephalic children. Child's Brain 1979; 5: 137- 
20. Kauffman, B., Weiss, M.H., Young, H.F., Nulsen, F.E.: Effects of prolonged cerebrospinal fluid shunting on the skull and brain. J Neurosurg 1973; 38: 288-297.

21. Keucher, T.R., Mealey, J.: Long-term results after ventriculoatrial and ventriculo-peritoneal shunting for infantile hydrocephalus. J Neurosurg 1979; 50: 179-186.

22. Kobayashi, A., Hashi, K.: Secondary spinal canal stenosis associated with long-term ventriculoperitoneal shunting. J Neurosurg 983; 59: 854-860.

23. Lain, C.H., Dubuisson, D.: Treatment of hemispheric collapse and herniation beneath the falx in a case of shunted hydrocephalus. Surg Neurol 1990; 33: 202-205.

24. Loop, J.E., Foltz, E.L.: Craniostenosis and diploic lamination following operation for hydrocephalus. Acta Radiol (Diagn) (Stockh) 1972; 13:8-13.

25. Lundar, T.: Shunt removal or replacement based on intracranial infusion tests. Child's Nerv Syst 1994; 10: 337339.

26. Martínez-Lage, J.F., Poza, M., López, F.: Arachnoid cyst as a complication of ventricular shunting. Child's Nerv Syst 1991; 7: 356-357.

27. Martínez-Lage, J.F., Alamo, L., Poza, M.: Raised intracranial pressure in minimal forms of craniosynostosis. Child's Nerv Syst 1999; 15: 11-16.

28. Novack, T.P., Jaines, H.J.: Migraine headaches in hydrocephalic children: a diagnostic dilemma. Child's Nerv Syst 1989; 5: 310-314.

29. Obana, W.G., Raskin, N.H., Logen, P.H., et al.: Antimigraine treatment for slit-ventricle syndrome. Neurosurgery 1990; 27: 760-763.

30. Oi, S., Matsumoto, S.: Slit ventricles as a cause of isolated ventricles after shunting. Child's Nerv Syst 1985; 1 : 189-193

31. Oi, S., Matsumoto, S.: Infantile hydrocephalus and the slit-ventricle syndrome in early infancy. Child's Nerv Syst 1987; 3: 145-150.

32. Oi, S., Kudo, H., Yamada, H., et al.: Hydromyelic hydrocephalus. J Neurosurg 1991; 74: 371-379.

33. Payner, T.D., Prenger, E., Berger, T.S., Crowe, K.R.: Acquired Chiari malformations: incidente, diagnosis and management. Neurosurgery 1994; 34: 429-434.

34. Reddy, K., Fewer, H.D., West, M., Hill, N.C.: Slit ventricle syndrome with aqueduct stenosis: third ventriculostomy as definitive treatment. Neurosurgery 1998; 23: 756-759.
2005; 16: 124-133

35. Rekate, H.L.: Classification of slit-ventricle syndromes using intracranial pressure monitoring. Pediatr Neurosurg 1993; 19: 15-20.

36. Rodríguez-Costa, T., Casas, C., Domingo, R., Miravete, M.T.M., Martínez-Lage, J.F., Puche, A.: Incidencia y evolución del colapso ventricular (slit-ventricle) en hidrocefalias posthemorrágicas neonatales y secundarias a mielomeningocele. Rev Neurol (Barc) 1994; 22: 167-170.

37. Rul-Lan Losada, G., Costa Clara, J.M., Lafuente, Millán, J., Fernández Alvarez, E.: Síndrome de ventrículos colapsados ("slit-ventricle"). A propósito de 8 observaciones. An Esp. Pediatr 1985; 22: 129-136.

38. Sainte-Rose, C.: Shunt obstruction: a preventable complicatíon? Pediatr Neurosurg 1995; 19: 156-164.

39. Salan, S., Sunder-Plassmann, M., Jellinger, K.: Late death in cases of treated internal hydrocephalus caused by encephalo-cranial disproportion. Mod Probl Pediatr 1977, 18: 149-151.

40. Serlo, W., Heikkinen, E., Saukkonen, A.L., Wendt, L.V.: Classification and management of the slit ventricle syndrome. Child's Nerv Syst 1985; 1: 194-199.

41. Serlo, W.: Experience with flow-regulated shunts (Orbis-Sigma valves) in cases of difficulty in managing hydrocephalus in children. Child's Nerv Syst 1995; 11: 166-169.

42. Sivalingan, S., Corkill, G.: Treatment of hydrocephalus and bilateral subdural effusions in a patient with closed sutures (case report). J Neurosurg 1976; 45: 447-448.

43. Steinbok, P., Berry, K.: lnversion of the cerebral mantle as a complication of ventricular shunting. J Neurosurg 1978; 49: 129-131.

44. Vassilyadi, M., Farmer, J.P., Montes, J.L.: Negative pressure hydrocephalus. J Neurosurg 1995; 83: 486-490.

45. Walsh, J.W., James, H.E.: Subtemporal craniectomy and elevation of shunt valve opening pressure in the management of small ventricle-induced cerebrospinal fluid shunt dysfunction. Neurosurgery 1982; 10: 698-703.

Martínez-Lage, J.F.; Pérez-Espejo, M.A.; Almagro, M.J.; Ros de San Pedro, J.; López, F.; Piqueras, C.; Tortosa, J.G.: Síndromes de hiperdrenaje de las válvulas en hidrocefalia infantil. Neurocirugía 2005; 16: 124-133.

Correspondencia postal: Juan F. Martínez-Lage. Servicio Regional de Neurocirugía. Hospital Universitario "Virgen de la Arrixaca". E-30120, El Palmar, Murcia 